\title{
Meson spectroscopy at COMPASS
}

\author{
Boris Grube ${ }^{1, \star}$ \\ for the COMPASS Collaboration \\ ${ }^{1}$ Institute for Hadronic Structure and Fundamental Symmetries, Physik-Department, Technische Universität \\ München
}

\begin{abstract}
The goal of the COMPASS experiment at CERN is to study the structure and dynamics of hadrons. The two-stage spectrometer used by the experiment has large acceptance and covers a wide kinematic range for charged as well as neutral particles and can therefore measure a wide range of reactions. The spectroscopy of light mesons is performed with negative (mostly $\left.\pi^{-}\right)$and positive $\left(p, \pi^{+}\right)$hadron beams with a momentum of $190 \mathrm{GeV} / c$. The light-meson spectrum is measured in different final states produced in diffractive dissociation reactions with squared four-momentum transfer $t$ to the target between 0.1 and $1.0(\mathrm{GeV} / c)^{2}$. The flagship channel is the $\pi^{-} \pi^{-} \pi^{+}$final state, for which COMPASS has recorded the currently world's largest data sample. These data not only allow to measure the properties of known resonances with high precision, but also to observe new states. Among these is a new axial-vector signal, the $a_{1}(1420)$, with unusual properties. Novel analysis techniques have been developed to extract also the amplitude of the $\pi^{-} \pi^{+}$subsystem as a function of $3 \pi$ mass from the data. The findings are confirmed by the analysis of the $\pi^{-} \pi^{0} \pi^{0}$ final state.
\end{abstract}

\section{Introduction}

The COMPASS experiment [1] has recorded large data sets of the diffractive dissociation reaction $\pi^{-}+p \rightarrow(3 \pi)^{-}+p_{\text {recoil }}$ using a $190 \mathrm{GeV} / c$ pion beam on a liquid-hydrogen target. In this process, the beam hadron is excited to some intermediate three-pion state $X^{-}$via $t$-channel Reggeon exchange with the target. At $190 \mathrm{GeV} / c$ beam momentum, Pomeron exchange is dominant. Diffractive reactions are known to exhibit a rich spectrum of intermediate states $X^{-}$and are a good place to search for states beyond the naive constituent-quark model. In the past, several candidates for so-called spin-exotic mesons, which have $J^{P C}$ quantum numbers that are forbidden in the non-relativistic quark model, have been reported in pion-induced diffraction $[2,3]$.

The scattering process is characterized by two kinematic variables: the squared total center-of-mass energy $s$, which is fixed by the beam energy, and the squared four-momentum transfer to the target $t=\left(p_{\text {beam }}-p_{X}\right)^{2}<0$. It is customary to use the reduced four-momentum transfer squared $t^{\prime} \equiv|t|-|t|_{\text {min }}$ instead of $t$, where $|t|_{\min }$ is the minimum value of $|t|$ for a given invariant mass of $X^{-}$. The analysis is performed in the range $0.1<t^{\prime}<1.0(\mathrm{GeV} / c)^{2}$.

In addition to the three final-state pions from the $X^{-}$decays, also the recoiling proton is measured. This helps to suppress backgrounds and ensures an exclusive measurement by applying energy and

^e-mail: bgrube@tum.de 

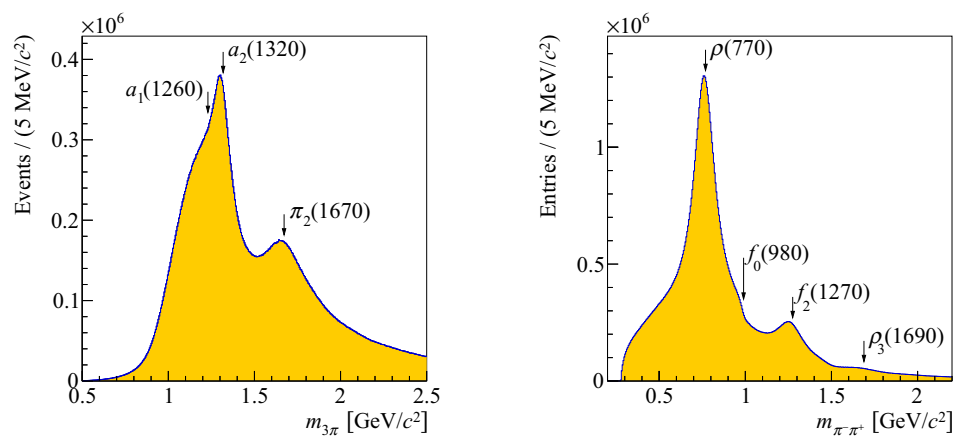

Figure 1. Left: $\pi^{-} \pi^{-} \pi^{+}$invariant mass spectrum in the analyzed range; Right: invariant mass distribution of the $\pi^{-} \pi^{+}$subsystem (two entries per event). Figures from Ref. [4].

momentum conservation in the event selection. After all selection cuts, the $3 \pi$ data samples consist of $46 \times 10^{6} \pi^{-} \pi^{-} \pi^{+}$and $3.5 \times 10^{6} \pi^{-} \pi^{0} \pi^{0}$ exclusive events in the analyzed kinematic region of three-pion mass, $0.5<m_{3 \pi}<2.5 \mathrm{GeV} / c^{2}$. Figure 1 shows the $\pi^{-} \pi^{-} \pi^{+}$invariant mass spectrum together with that of the $\pi^{-} \pi^{+}$subsystem. The known pattern of resonances $a_{1}(1260), a_{2}(1320)$, and $\pi_{2}(1670)$ is seen in the $3 \pi$ system along with $\rho(770), f_{0}(980), f_{2}(1270)$, and $\rho_{3}(1690)$ in the $\pi^{-} \pi^{+}$subsystem.

\section{Partial-wave decomposition}

In order to disentangle the different contributing intermediate states $X^{-}$, a partial-wave analysis (PWA) is performed. The PWA of the $(3 \pi)^{-}$final states is based on the isobar model, which assumes that the $X^{-}$decays first into an intermediate resonance, which is called the isobar, and a "bachelor" pion $\left(\pi^{-}\right.$ for the $\pi^{-} \pi^{-} \pi^{+}$final state; $\pi^{-}$or $\pi^{0}$ for $\pi^{-} \pi^{0} \pi^{0}$ ). In a second step, the isobar decays into two pions. In accordance with the $\pi^{-} \pi^{+}$invariant mass spectrum shown in Fig. 1 right and with analyses by previous experiments, we include $[\pi \pi]_{S}, \rho(770), f_{0}(980), f_{2}(1270), f_{0}(1500)$, and $\rho_{3}(1690)$ as isobars into the fit model. Here, $[\pi \pi]_{S}$ represents the broad component of the $\pi \pi S$-wave. Based on the six isobars, we have constructed a set of partial waves that consists of 88 waves in total, including one non-interfering isotropic wave representing three uncorrelated pions. This constitues the largest wave set ever used in an analysis of the $3 \pi$ final state. The partial-wave decomposition is performed in narrow bins of the $3 \pi$ invariant mass. Since the data show a complicated correlation of the $m_{3 \pi}$ and $t^{\prime}$ spectra, each $m_{3 \pi}$ bin is further subdivided into non-equidistant bins in the four-momentum transfer $t^{\prime}$. For the $\pi^{-} \pi^{-} \pi^{+}$channel 11 bins are used, for the $\pi^{-} \pi^{0} \pi^{0}$ final state 8 bins. With this additional binning in $t^{\prime}$, the dependence of the individual partial-wave amplitudes on the four-momentum transfer can be studied in detail. The details of the analysis model are described in Ref. [4].

The partial-wave amplitudes are extracted from the data as a function of $m_{3 \pi}$ and $t^{\prime}$ by fitting the five-dimensional kinematic distributions of the outgoing three pions. The amplitudes do contain information not only about the partial-wave intensities, but also about the relative phases of the partial waves. The latter are crucial for resonance extraction. The three-pion partial waves are defined by the quantum numbers of the $X^{-}$(spin $J$, parity $P, C$-parity, absolute value $M$ of the spin projection), the naturality $\varepsilon= \pm 1$ of the exchange particle, the isobar, and the orbital angular momentum $L$ between the isobar and the bachelor pion. These quantities are summarized in the partial-wave notation $J^{P C}$ $M^{\varepsilon}$ [isobar] $\pi L$. Since at the used beam energies Pomeron exchange is dominant, 80 of the 88 partial waves in the model have $\varepsilon=+1$. 

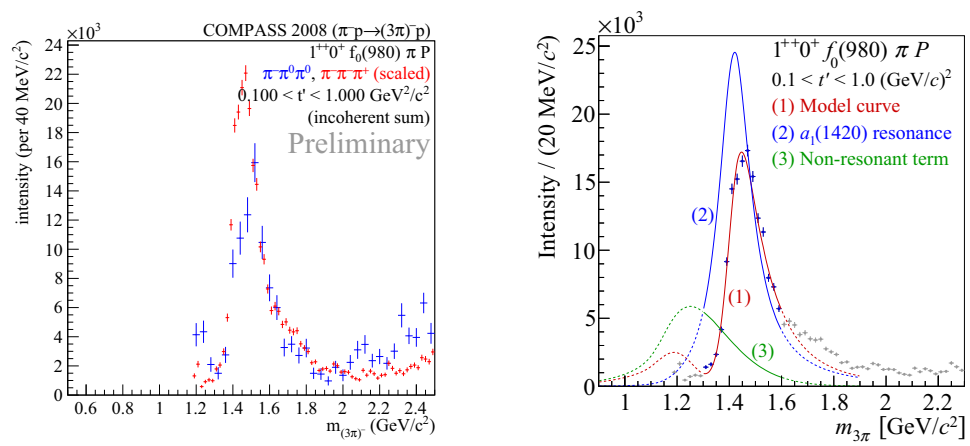

Figure 2. Left: Intensity of the $1^{++} 0^{+} f_{0}(980) \pi P$ wave summed over all $t^{\prime}$ bins for the $\pi^{-} \pi^{0} \pi^{0}$ (blue) and the $\pi^{-} \pi^{-} \pi^{+}$(red, scaled to the intensity integral of the $\pi^{-} \pi^{0} \pi^{0}$ channel) final states. Right: Result of a resonance-model fit to the $\pi^{-} \pi^{-} \pi^{+}$data [5]. The data points correspond to the red points in the left figure.
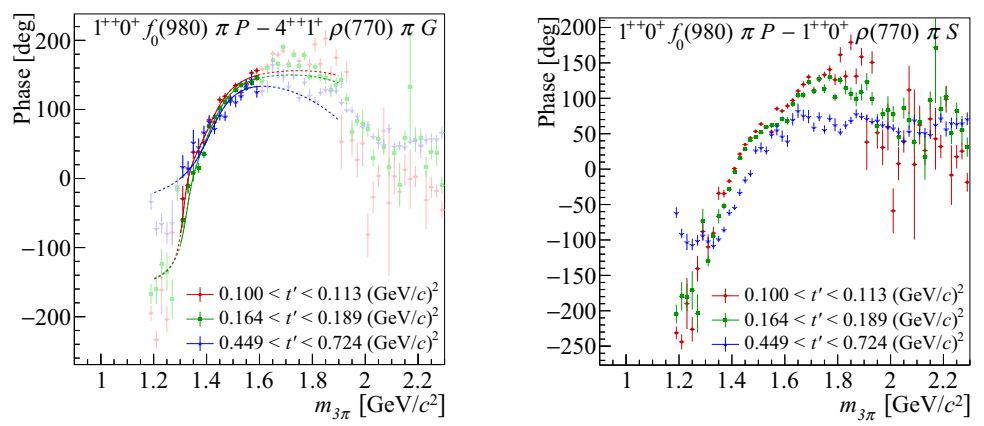

Figure 3. Examples for relative phases of the $1^{++} 0^{+} f_{0}(980) \pi P$ wave with respect to the $4^{++} 1^{+} \rho(770) \pi G$ (left) and the $1^{++} 0^{+} \rho(770) \pi S$ wave (right). The phases are shown for three different $t^{\prime}$ regions indicated by the color. Figures from Ref. [5].

\subsection{The $a_{1}(1420)$}

A surprising find in the COMPASS data is a pronounced narrow peak at about $1.4 \mathrm{GeV} / c^{2}$ in the $1^{++}$ $0^{+} f_{0}(980) \pi P$ wave (see Fig. 2). The peak is observed with similar shape in the $\pi^{-} \pi^{-} \pi^{+}$and $\pi^{-} \pi^{0} \pi^{0}$ channels and is robust against variations of the PWA model. In addition to the peak in the partial-wave intensity, rapid phase variations with respect to most waves are observed in the $1.4 \mathrm{GeV} / c^{2}$ region (see Fig. 3). The phase motion as well as the peak shape change only little with $t^{\prime}$.

In order to test the compatibility of the signal with a Breit-Wigner resonance, a resonance-model fit was performed using a novel method, where the intensities and relative phases of three waves $\left[1^{++}\right.$ $0^{+} f_{0}(980) \pi P, 2^{++} 1^{+} \rho(770) \pi D$, and $4^{++} 1^{+} \rho(770) \pi G$ ] were fit simultaneously in all $11 t^{\prime}$ bins [5]. Forcing the resonance parameters to be the same across all $t^{\prime}$ bins leads to an improved separation of resonant and non-resonant contribution as compared to previous analyses that did not incorporate the $t^{\prime}$ information. The Breit-Wigner model describes the peak in the $1^{++} 0^{+} f_{0}(980) \pi P$ wave well and yields a mass of $m_{0}=\left(1414_{-13}^{+15}\right) \mathrm{MeV} / c^{2}$ and a width of $\Gamma_{0}=\left(153_{-23}^{+8}\right) \mathrm{MeV} / c^{2}$ for the $a_{1}(1420)$. Due to the high statistical precision of the data, the uncertainties are dominated by systematic effects. 
The $a_{1}(1420)$ signal is remarkable in many ways. It appears in a mass region that is well studied since decades. However, previous experiments were unable to see the peak, because it contributes only $0.25 \%$ to the total intensity. The $a_{1}(1420)$ is very close in mass to the $1^{++}$ground state, the $a_{1}(1260)$. But it has a much smaller width than the $a_{1}(1260)$. The $a_{1}(1420)$ peak is seen only in the $f_{0}(980) \pi$ decay mode of the $1^{++}$waves and lies suspiciously close to the $K \bar{K}^{*}(892)$ threshold.

The nature of the $a_{1}(1420)$ is still unclear and several interpretations were proposed. It could be the isospin partner to the $f_{1}(1420)$. It was also described as a two-quark-tetraquark mixed state [6] and a tetraquark with mixed flavor symmetry [7]. Other models do not require an additional resonance: the authors of Refs. [8,9] propose resonant re-scattering corrections in the Deck process as an explanation, whereas Ref. [10] suggests a branching point in the triangular rescattering diagram for $a_{1}(1260) \rightarrow K \bar{K}^{*}(892) \rightarrow K \bar{K} \pi \rightarrow f_{0}(980) \pi$. The results of the latter calculation were confirmed by the authors of Ref. [11]. Triangle singularities were also proposed as an explanation for the narrow $\eta(1405)$ [12, 13], for some of the near-threshold $X Y Z$ heavy-quark states (see e.g. Ref. [14]), and for the pentaquark candidate $P_{c}(4450)$ recently found by $\operatorname{LHCb}[15,16]$. More detailed studies are needed in order to distinguish between the different models for the $a_{1}(1420)$.

\subsection{Extraction of $\pi \pi S$-wave isobar amplitudes from data}

The PWA of the $3 \pi$ system is based on the isobar model, where fixed amplitudes are used for the description of the $\pi^{-} \pi^{+}$intermediate states. However, we cannot exclude that the fit results are biased by the employed isobar parametrizations. This is true in particular for the isoscalar $J^{P C}=0^{++}$isobars. In the PWA model, a broad $\pi \pi S$-wave component is used, the parametrization of which is extracted from $\pi \pi S$-wave elastic-scattering data [17]. In addition, the $f_{0}(980)$, described by a Flatté form [18], and the $f_{0}(1500)$, parametrized by a relativistic Breit-Wigner amplitude, are included as isobars. In order to study possible bias due to these parametrizations and to ensure that the observed $a_{1}(1420)$ signal is truly related to the narrow $f_{0}(980)$, a novel analysis method inspired by Ref. [19] was developed [4]. In this so-called freed-isobar analysis, the three fixed parametrizations for the $0^{++}$isobar amplitudes are replaced by a set of piecewise constant complex-valued functions that fully cover the allowed two-pion mass range. This way the whole $0^{++}$isobar amplitude is extracted as a function of the $3 \pi$ mass. In contrast to the conventional isobar approach, which uses the same isobar parametrization in different partial waves, the freed-isobar method permits different isobar amplitudes for different intermediate states $X^{-}$. A more detailed description of the analysis method can be found in Ref. [4].

The freed-isobar method leads to a reduced model bias and gives additional information about the $\pi^{-} \pi^{+}$subsystem at the cost of a considerable increase in the number of free parameters in the PWA fit. Thus, even for large data sets, the freed-isobar approach can only be applied to a subset of partial waves. We performed a freed-isobar PWA, where the fixed parametrizations of the broad $\pi \pi S$-wave component, of the $f_{0}(980)$, and of the $f_{0}(1500)$ were replaced by piece-wise constant isobar amplitudes for the $3 \pi$ partial waves $0^{-+} 0^{+}[\pi \pi]_{0^{++}} \pi S, 1^{++} 0^{+}[\pi \pi]_{0^{++}} \pi P$, and $2^{-+} 0^{+}[\pi \pi]_{0^{++}} \pi D$. Figure 4 left shows the two-dimensional intensity distribution of the $1^{++} 0^{+}[\pi \pi]_{0^{++}} \pi P$ wave as a function of $m_{\pi^{-} \pi^{+}}$and $m_{3 \pi}$. The distribution exhibits a broad maximum around $m_{3 \pi}=1.2 \mathrm{GeV} / c^{2}$ and between 0.6 and $0.8 \mathrm{GeV} / c^{2}$ in $m_{\pi^{-} \pi^{+}}$, which shows a pronounced $t^{\prime}$ dependence and therefore is probably mainly of non-resonant origin. A smaller peak is observed in the $f_{0}(980)$ region at $m_{3 \pi} \approx 1.4 \mathrm{GeV} / c^{2}$. This peak is more obvious in Fig. 4 center, which shows the intensity distribution summed over the two-pion mass interval around the $f_{0}(980)$ as indicated by the pair of horizontal dashed lines in Fig. 4 left. The peak is similar in position and shape to the $a_{1}(1420)$ peak in the $1^{++} 0^{+} f_{0}(980) \pi P$ wave (cf. Fig. 2). The resonant nature of the $f_{0}(980)$ becomes apparent in Fig. 4 right, which shows the $m_{\pi^{-} \pi^{+}}$dependence of the extracted amplitude at the $a_{1}(1420)$ peak in form of an Argand diagram. The phase is measured with respect to the $1^{++} 0^{+} \rho(770) \pi S$ wave. The $f_{0}(980)$ contribution shows up as a semicircle-like structure 

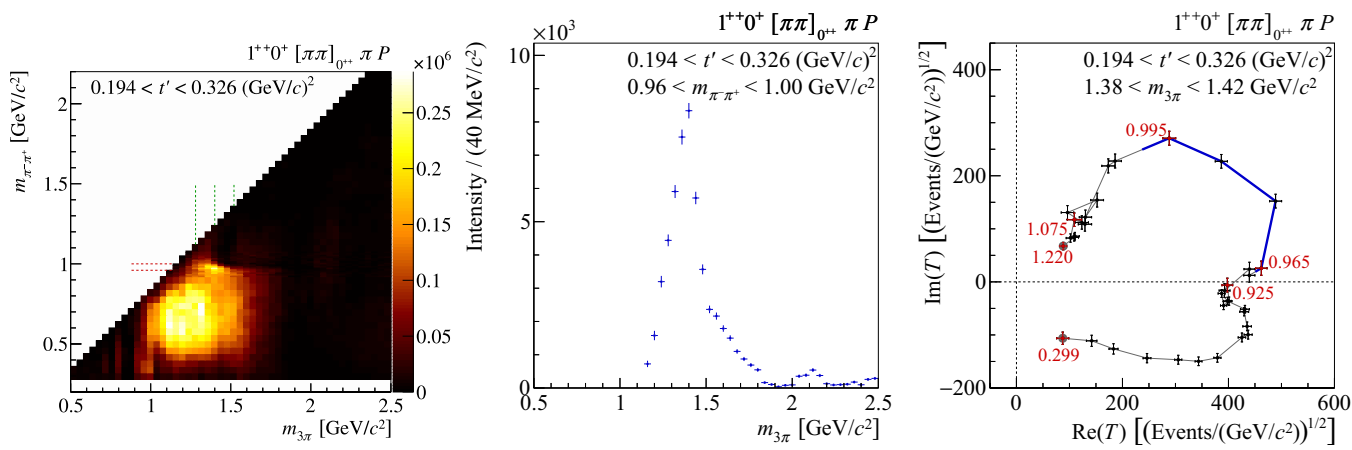

Figure 4. Left: Intensity of the $\pi \pi S$-wave component of the $J^{P C} M^{\varepsilon}=1^{++} 0^{+}$partial wave resulting from the freed-isobar fit as a function of $m_{\pi^{-} \pi^{+}}$and $m_{3 \pi}$. Center: intensity as a function of $m_{3 \pi}$ summed over the $m_{\pi^{-} \pi^{+}}$ interval around the $f_{0}(980)$ indicated by the pair of horizontal dashed lines in the top figure. Right: Argand diagram representing the $m_{\pi^{-} \pi^{+}}$dependence of the partial-wave amplitude for the $3 \pi$ mass bin at the $a_{1}(1420)$ measured with respect to the $1^{++} 0^{+} \rho(770) \pi S$ wave. Figures from Ref. [4].

(highlighted by the blue line) with a shifted origin. This demonstrates that the observed $a_{1}(1420)$ signal in the $f_{0}(980) \pi$ decay mode is not an artifact of the $0^{++}$isobar parametrizations used in the conventional PWA method. More results of the freed-isobar PWA are discussed in Ref. [4].

\subsection{The $J^{P C}=1^{-+}$spin-exotic wave}

The 88-wave model also contains waves with exotic $J^{P C}$ quantum numbers, that are forbidden in the non-relativistic quark model. The most interesting of these waves is the $1^{-+} 1^{+} \rho(770) \pi P$ wave, which contributes less than $1 \%$ to the total intensity. Previous analyses claimed a resonance, the $\pi_{1}(1600)$, at about $1.6 \mathrm{GeV} / c^{2}$ in this channel $[20,21]$. Figure 5 left shows the intensity sum over all $t^{\prime}$ bins of this partial wave for the two final states $\left(\pi^{-} \pi^{-} \pi^{+}\right.$in red, $\pi^{-} \pi^{0} \pi^{0}$ in blue). The two distributions are scaled to have the same integral. Both decay channels are in fair agreement and exhibit a broad enhancement extending from about 1.0 to $1.8 \mathrm{GeV} / c^{2}$ in $m_{3 \pi}$. In the 1.0 to $1.2 \mathrm{GeV} / c^{2}$ mass range, the intensity depends strongly on the details of the fit model. Peak-like structures in this region are probably due to cross talk induced by imperfections of the applied PWA model.

A remarkable change of the shape of the intensity spectrum of the $1^{-+} 1^{+} \rho(770) \pi P$ wave with $t^{\prime}$ is observed (see dark blue points in Fig. 5 center and right). At values of $t^{\prime}$ below about $0.3(\mathrm{GeV} / c)^{2}$, we observe no indication of a resonance peak around $m_{3 \pi}=1.6 \mathrm{GeV} / \mathrm{c}^{2}$, where we would expect the $\pi_{1}(1600)$. However, for the $t^{\prime}$ bins in the interval $0.449<t^{\prime}<1.000(\mathrm{GeV} / c)^{2}$, the observed intensities exhibit a very different shape as compared to the low- $t^{\prime}$ region, with a peak structure emerging at about $1.6 \mathrm{GeV} / c^{2}$ and the intensity at lower masses disappearing rapidly with increasing $t^{\prime}$. This is in contrast to clean resonance signals like the $a_{2}(1320)$ in the $2^{++} 1^{+} \rho(770) \pi D$ wave, which, as expected, do not change their shape with $t^{\prime}$. The observed $t^{\prime}$ behavior of the $1^{-+}$intensity is therefore a strong indication that non-resonant contributions play a dominant role.

It is believed that the non-resonant contribution in the $1^{-+}$wave originates predominantly from the Deck effect, in which the incoming beam pion dissociates into the isobar and an off-shell pion that scatters off the target proton to become on-shell [22]. As a first step towards a better understanding of the non-resonant contribution, Monte-Carlo data were generated that are distributed according to a model of the Deck effect. The model employed here is very similar to that used in Ref. [23]. The 

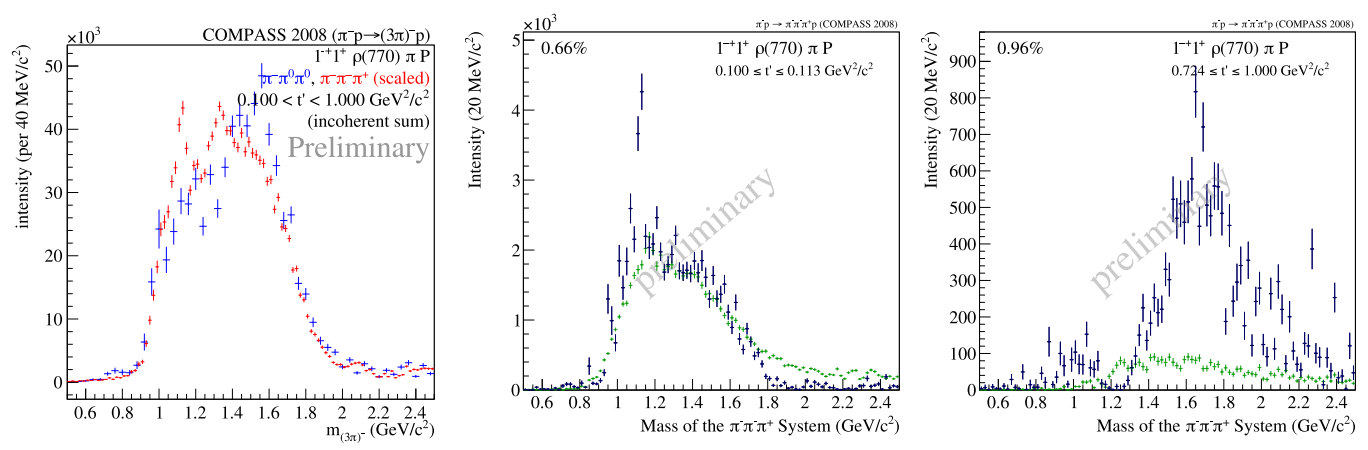

Figure 5. Intensity of the $1^{-+} 1^{+} \rho(770) \pi P$ wave. Left: summed over all $t^{\prime}$ bins for the $\pi^{-} \pi^{-} \pi^{+}$(red) and the $\pi^{-} \pi^{0} \pi^{0}$ (blue) final state. Center and right panels show the intensity for the $\pi^{-} \pi^{-} \pi^{+}$final state (dark blue) in different regions of $t^{\prime}$ (center: low $t^{\prime}$; right: high $t^{\prime}$ ). The partial-wave projections of Monte-Carlo data generated according to a model of the Deck effect are overlaid in green.

partial-wave projection of these Monte Carlo data is shown as green points in Fig. 5 center and right. In order to compare the intensities of real data and the Deck-model pseudo data, the Monte Carlo data are scaled to the $t^{\prime}$-summed intensity of the $1^{-+}$wave as observed in real data. At values of $t^{\prime}$ below about $0.3(\mathrm{GeV} / c)^{2}$, the intensity distributions of real data and Deck Monte Carlo exhibit strong similarities suggesting that the observed intensity might be saturated by the Deck effect. Starting from $t^{\prime} \approx 0.4(\mathrm{GeV} / c)^{2}$, the spectral shapes for Deck pseudo data and real data deviate from each other with the differences increasing towards larger values of $t^{\prime}$. This leaves room for a potential resonance signal. It should be noted, however, that the Deck pseudo data contain no resonant contributions. Therefore, potential interference effects between the resonant and non-resonant amplitudes cannot be assessed in this simple approach.

\section{Acknowledgements}

This work was supported by the BMBF, the Maier-Leibnitz-Laboratorium (MLL), the DFG Cluster of Excellence Exc153 "Origin and Structure of the Universe", and the computing facilities of the Computational Center for Particle and Astrophysics (C2PAP).

\section{References}

[1] P. Abbon et al. (COMPASS Collaboration), Nucl. Instrum. Methods Phys. Res., Sect. A 779, 69 (2015)

[2] C. Meyer et al., Phys. Rev. C 82, 025208 (2010)

[3] E. Klempt et al., Phys. Rept. 454, 1 (2007)

[4] C. Adolph et al. (COMPASS Collaboration), arXiv:1509.00992, submitted to Phys. Rev. D (2015)

[5] C. Adolph et al. (COMPASS Collaboration), Phys. Rev. Lett. 115, 082001 (2015)

[6] Z.G. Wang, arXiv:1401.1134 (2014)

[7] H.X. Chen et al., Phys. Rev. D 91, 094022 (2015)

[8] J.L. Basdevant, E.L. Berger, Phys. Rev. Lett. 114, 192001 (2015)

[9] J.L. Basdevant, E.L. Berger, arXiv:1501.04643 (2015) 
[10] M. Mikhasenko et al., Phys. Rev. D 91, 094015 (2015)

[11] F. Aceti, L.R. Dai, E. Oset, arXiv:1606.06893 (2016)

[12] J.J. Wu, X.H. Liu, Q. Zhao, B.S. Zou, Phys. Rev. Lett. 108, 081803 (2012)

[13] X.G. Wu, J.J. Wu, Q. Zhao, B.S. Zou, Phys. Rev. D 87, 014023 (2013)

[14] A.P. Szczepaniak, Phys. Lett. B 747, 410 (2015)

[15] R. Aaij et al. (LHCb Collaboration), Phys. Rev. Lett. 115, 072001 (2015)

[16] F.K. Guo, U.G. Meißner, W. Wang, Z. Yang, Phys. Rev. D 92, 071502 (2015)

[17] K.L. Au, D. Morgan, M.R. Pennington, Phys. Rev. D 35, 1633 (1987)

[18] M. Ablikim et al. (BES Collaboration), Phys. Lett. B 607, 243 (2005)

[19] E.M. Aitala et al. (E791 Collaboration), Phys. Rev. D 73, 032004 (2006)

[20] S.U. Chung et al. (E852 Collaboration), Phys. Rev. D 60, 092001 (1999)

[21] M. Alekseev et al. (COMPASS Collaboration), Phys. Rev. Lett. 104, 241803 (2010)

[22] R.T. Deck, Phys. Rev. Lett. 13, 169 (1964)

[23] C. Daum et al. (ACCMOR Collaboration), Nucl. Phys. B 182, 269 (1981) 